\title{
Resection in Survey
}

\section{Author(s): G. T. McCaw}

Source: The Geographical Journal, Vol. 52, No. 2 (Aug., 1918), pp. 105-123

Published by: geographicalj

Stable URL: http://www.jstor.org/stable/1779558

Accessed: 18-06-2016 10:29 UTC

\section{Your use of the JSTOR archive indicates your acceptance of the Terms \& Conditions of Use, available at}

http://about.jstor.org/terms

JSTOR is a not-for-profit service that helps scholars, researchers, and students discover, use, and build upon a wide range of content in a trusted digital archive. We use information technology and tools to increase productivity and facilitate new forms of scholarship. For more information about JSTOR, please contact support@jstor.org.

The Royal Geographical Society (with the Institute of British Geographers), Wiley are collaborating with JSTOR to digitize, preserve and extend access to The Geographical Journal 
was a most remarkable performance. Sir Ernest Satow was not a man to advertise himself, but I am glad to have this opportunity of testifying to the enormous work he has done. As for Mr. MacLeod, I can only say that it was a great pleasure to me to see him here after twenty-five years. I know the work he did in $\mathrm{Fez}$; I was a recipient of his hospitality; and the few days I spent there were most pleasant. I have heard of him from time to time ever since, and have witnessed the very great work he has done, and the selfabnegation he has shown in remaining in Morocco and carrying out the work of caring for British interests for thirty years.

The PRESIDENT: I have regretted all my life that I have never been anywhere near Morocco. There is a point which I think many of you will recognize at once as indicative of the extraordinary progress which the French have made there. Not so very long ago a lecturer here told us of his journey southeast from Mogador to the Atlas range, where he found a very beautiful valley at the foot of the Atlas Mountains, and was entertained royally by a local sheikh and his village people. But after he left he found that a very ingenious plot had been made to murder him, and he heard that the sheikh who had entertained him and all the villagers who had assisted were massacred by the people from the hills. It was eventually proved, beyond the shadow of a doubt, that all this was due to German intrigue and German money. That was in the year I9I -just those six or seven years ago when the French began their reclamation of Morocco. One would like to ask what has become of German interests in Morocco. It seems to me that we may possibly be able to congratulate ourselves that the occupation of the French there, their marvellous energy, their remarkable achievements, have completely superseded them. I rather regret that in the course of the lecture Mr. MacLeod has not been able to indulge in a few more personalities. Many of us would like to know something about that very picturesque old rascal Rasuli, the most powerful Arab in Morocco, who will, no doubt, in a comparatively short time, figure in war-time history. You will all agree that we have enjoyed a most interesting paper, and I will ask you to join in a formal vote of thanks to Mr. MacLeod for his address.

\section{RESECTION IN SURVEY}

\section{G. T. McCAW, M.A., F.R.A.S.}

\section{Read at the Afternoon Meeting of the Society, I 5 April I9I8.}

$\mathrm{R}^{\mathrm{E}}$ ESECTION in survey is a method of observation which is opposed to Intersection. An outpoint, itself unoccupied by an observer, which is observed from fixed stations, is known as an "Intersected Point." An outpoint which is occupied and whose position is determined by angular measures made to fixed stations which are not themselves occupied for the purpose of determining the position of such a point, is known as a "Resected point." If $\mathrm{A}$ is a fixed station and $\mathrm{P}$ an outpoint, nonreciprocal observation from $\mathrm{A}$ to $\mathrm{P}$ implies intersection; from $\mathrm{P}$ to $\mathrm{A}$, resection.

This method of interpolating positions possesses considerable advantages from the point of view of speed, economy, and convenience. In 
certain kinds of work its advantages are so overwhelming that practically no other course is open to the surveyor; it is in consequence most commonly employed by topographers, including hydrographers. It is safe to say that a great, if not the greater, part of our knowledge of the superficial features of the Earth is due to the almost universal employment of this method for the construction of maps and charts. When a country has reached that high degree of development which is characteristic of our home lands, that is to say, when the country is covered by an accurate cadastral survey with which topography is united, the employment of the method is no longer necessary ; but it is far otherwise in undeveloped or less highly developed countries. Here the mere question of economy renders its employment necessary. Here the enormous saving of time rendered possible by a method of fixing one's position by observations made in situ is usually the consideration which alone enables the State unsupported to carry out in the early stages of its development a general delineation of its superficial features.

To understand the reason for this great saving in expense it is necessary to remember that main stations are usually situated on the highest positions, and, in consequence, most frequently in areas not easily accessible; and even secondary stations are not always easy of access. But much more important than this consideration is the fact that these stations are necessarily limited in number, and from them it would be impossible to map all the detail in the district. Topography obviously involves an immense amount of detailed work, and the smaller features can be taken in only if the number of topographical working sites is greatly multiplied; more especially is this the case in countries greatly broken.

To carry out such a survey it is necessary in all cases to have a minor triangulation, consisting of secondary points. These triangles may again be broken up so as to give a sufficient number of tertiary stations to form the fundamental positions for the topographer. The tertiary stations may sometimes be fixed by trigonometrical interpolation; that is to say, by employing the method of resection in conjunction with a theodolite. In very difficult circumstances, such as in the region of the Himalayas, Hindu Kush, and Pamir, trigonometrical interpolation may be perhaps the only possible means of fixing the position of the higher peaks. In general, however, with the fundamental positions of his survey all fixed by theodolite with considerable accuracy, the topographer finds that the plane-table or camera gives sufficiently accurate location of detail, with enormous saving of expense.

In speaking of the accuracy of the method, one must distinguish between the accuracy inherent in the method and the accuracy of the method in conjunction with a given instrument. There is, for example, a profound difference between the results obtained by a plane-table and, say, a good 6-inch theodolite. While it is to be remembered that where one 
instrument is useful the other is useless, and vice vers $\hat{a}$, this paper regards the subject more from the point of view of the worker with the theodolite; where the occasion suggests it, reference will be made to the plane-table, in connection with which the method has received already the full treatment which the almost universal employment of this Mensula pratoriana demands. Further, since high accuracy is not required from the planetable, any discussion would be foreign to those parts of the present paper which are concerned with the computation of error. On the other hand, in so far as the present investigation indicates positions which may be obtained geometrically, it applies as much to the plane-table as to the theodolite.

The inherent accuracy of the method is not so great as that of intersection. Roughly speaking, the displacement of a resected point remote from the danger circle varies as the square of the distances from the fixed stations, while the displacement of an intersected point consequent on errors in the angles varies as the distances. Nevertheless, the author is of opinion that the limitation of resection is more a handicap of position than a consequence of inherent and unavoidable inaccuracy. It ought to be remembered in comparing the two methods that, in the case of two measured angles, intersection implies observations from two fixed stations only, while resection demands three, thus securing some compensation towards increased accuracy. If the resected part is well placed, there need not be any great difference between the errors in the two methods. This is proved by the following considerations. Suppose that a point $\mathrm{P}$ is fixed by resection from three stations $\mathrm{A}, \mathrm{B}, \mathrm{C}$, and again by intersection from $\mathrm{A}$ and $\mathrm{B}$. Suppose also that the sides $\mathrm{BC}, \mathrm{CA}$, are $a, b$ respectively, $\mathrm{PA}=p, \mathrm{~PB}=q, \mathrm{PC}=r$, and that the angle $\mathrm{APB}$ at $\mathrm{P}$ is $\gamma$, while that of the fixed triangle at $\mathrm{C}$ is $C$. Then if the mean square error of an angle be $\mu_{c}^{\prime \prime}$, the mean square error of the position of $\mathrm{P}$ is

By resection . $\pm \mu_{a} r \operatorname{cosec}(\gamma-C) \sin \mathrm{I}^{\prime \prime} \sqrt{ }\left(q^{2} / a^{2}+p^{2} / b^{2}\right)$,

By intersection . $\pm \mu_{a} \operatorname{cosec} \gamma \sin \mathrm{x}^{\prime \prime} \sqrt{ }\left(p^{2}+q^{2}\right)$.

The similarity between these two formulæ is striking evidence of the justness of the contention here put forward. If $q$ is comparable in length with $a$ and $p$ with $b$, and $\gamma-C$ is not near $0^{\circ}$ or $180^{\circ}$, the two results need not greatly differ. It must be remembered, too, that, there is a limitation of position in the case of intersected points also; the triangles must not be too badly conditioned. We see this very clearly from the formulæ, each of which includes an element of weakness; without involving errors theoretically infinite, $\gamma$ can be no more equal to $0^{\circ}$ or $180^{\circ}$ in intersection than $\gamma-C$ can have the same values in resection. It is quite possible to have an excellent position for resection from three stations where intersection from two of them would be useless. In summing up, then, it may be said that, while intersection is in general the better method, there is a limited field within which resection will 
afford quite comparable results - a fact not generally known. If we are to consider intersection from three, we ought equally to consider resection from four fixed stations, the number of measured angles being the same in both cases. To determine means of estimating a priori the precision of resection, and alternately the possibility of utilizing the method in given circumstances, is one of the chief objects of this paper.

Owing to the limitation mentioned resection is never employed in purely primary survey. If used by geodetic surveyors, as may rarely occur, it is applied to the location of secondary points, and even then the observations are multiplied and reduced by the method of least squares, thus arriving at an accuracy, as proved by the computed errors, comparable with that of intersection. In cadastral survey the method is not permissible, simply because it is not necessary. This should be an absolute rule for governing internal surveys; but there may be something to be said for employing the method, under strictly defined regulations, for the location, on provisional maps, of boundary points of estates in undeveloped countries. In various places the connection of these with main or secondary stations would entail heavy expense, which should not be demanded of a single occupier, the State as a whole being responsible for these connections. But in this case it appears to be clearly a mistake to make such location dependent on plotment alone; as computation is by no means operose, it would seem that it should be required, in conjunction with sufficiently precise instrumentation. Such computation, moreover, will often provide a valuable control on the accuracy of the internal survey.

With these preliminary remarks regarding resection-a word used by surveyors and surgeons in two widely different senses, though the root idea of "cutting back" is common to both-we shall proceed to consider the various problems which are likely to arise. Of these we have already referred to the error in case of the first and most common problem; there are, however, about half a dozen in all which are of value. They may be divided into single-point and double-point problems, since multiple-point problems are very unlikely to arise unless in combination with intersection. They may also be divided into problems which are directly applicable to the plane-table and those which cannot be employed therewith without involving rather complex figures which necessitate readjustment. The plane-table is not, however, limited to the single-point problems : there are double-point problems for which it is eminently suitable. In general, however, double-point problems are of greater value for trigonometrical interpolation. They may again be divided into classes according as they involve two, three or four fixed stations, thus becoming problems of the triangle (three fixed stations) or quadrilateral (two or four fixed stations). Lastly, they may be classed according as they involve pure resection, resection mixed with intersection, or resection allied with measurement of vertical angles. These divisions are of course overlapping. 


\section{The First Problem.}

The first and most common problem is illustrated in Fig. I. A, B, C are three fixed points-possibly main or secondary stations on hills or mountains-and $\mathrm{P}$ is a point-possibly in a plain-whose position it is desired to fix without visiting $A, B$, or $C$. It is in general sufficient to measure two of the angles at $\mathrm{P}$, marked $\alpha, \beta, \gamma$. Before proceeding to an analysis of this problem, it will not be out of place to make a few remarks as to its history, which is apparently unknown in this country.

It is to Holland that we are indebted for the most complete historical investigation, in which the names of Dr. J. D. van der Plaats and the well-known geodesist Prof. J. A. C. Oudemans are conspicuous. According to Dr. van der Plaats the problem was probably well known to the successors of Eucleides in the great Alexandrian School, being an almost obvious corollary of Euclid III. 2 I. Indeed

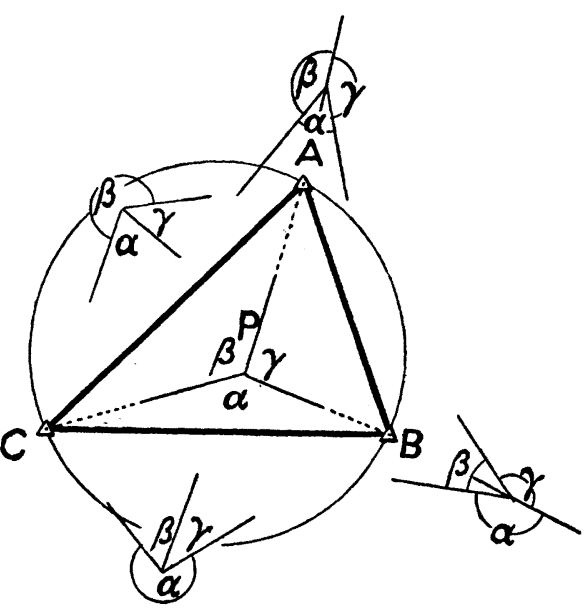

FIG. I. one might add that it would be no more absurd to think that the famous proposition followed from an attempt on the part of some old surveyor in Mesopotamia or Egypt to solve this or some cognate problem than to think that Eucleides himself was the originator of all the propositions which are so rightly identified with his name. The same authority shows that the problem was used for an astronomical purpose by Hipparchus (circa 150 B.c.), while Oudemans finds an astronomical application in Ptolemy also (circa 140 A.D.).

Nothing further is known for fifteen centuries until its rediscovery by the famous Willebrord Snel van Roien (1580-1626), who was the first, so far as is known, to employ the method for a geodetic object, some time before 1617 . In this year the Batavian Eratosthenes, thus self-styled with pardonable pride, published his epoch-making work, marking the dawn of modern survey. A few remarks regarding the solution by Snellius-he is generally known by the Latinized form of the name-will not be out of place.

By Fig. 2 which follows we can indicate his proof. Wishing to fix the position of his house at Leyden, at $\mathrm{P}$ say, he measures the angles $\alpha, \beta$ subtended by three points $\mathrm{A}, \mathrm{B}, \mathrm{C}$, fixed by his triangulation. In solving, he makes use of the triangle $\mathrm{O}_{1} \mathrm{CO}_{2}$, connecting the centres of the usual 
circles with that apex which they intersect in common. The angle $\mathrm{O}_{1} \mathrm{CO}_{2}$ is equal to $C+\alpha+\beta-180^{\circ}, C$ being the angle of the triangle, and is therefore known. Now, the radii $\mathrm{CO}_{1}, \mathrm{CO}_{2}$ of the circles are known, being equal to $\frac{1}{2} a \operatorname{cosec} a, \frac{1}{2} b \operatorname{cosec} \beta$ respectively, $a, b$ being the sides $\mathrm{BC}, \mathrm{CA}$ of the fixed triangle. Hence in the triangle $\mathrm{O}_{1} \mathrm{CO}_{2}$ we have two sides and the included angle, from which the base angles $\mathrm{CO}_{1} \mathrm{O}_{2}$,

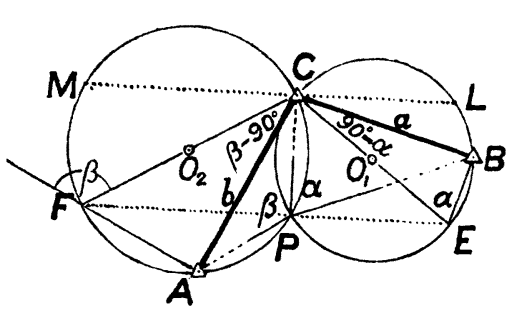

FIG. 2. $\mathrm{CO}_{2} \mathrm{O}_{1}$ can be computed. Then evidently $\mathrm{CP}={ }_{2} \mathrm{CO}_{1}$ sin $\mathrm{CO}_{1} \mathrm{O}_{2}$ $={ }_{2} \mathrm{CO}_{2} \sin \mathrm{CO}_{2} \mathrm{O}_{1}$. Since the angle $\mathrm{CO}_{1} \mathrm{O}_{2}=\mathrm{CEP}=\mathrm{CBP}$, and the angle $\mathrm{CO}_{2} \mathrm{O}_{1}=\mathrm{CAP}$, the other two distances $\mathrm{AP}, \mathrm{BP}$ follow by the rule of sines, applied in the triangles $\mathrm{BCP}, \mathrm{CAP}$.

It is no excessive tribute to the contributions made by Snel to the knowledge of our Earth to follow the Dutch of Holland and South Africa and name $P$ the Snellius point.

In 1624 , Schickhart, who conducted the second European triangulation in the Swabian Jura (Rauhe Alps, near Tübingen) from 1624-35, in a letter to Kepler mentions the problem and the ordinary solution by the aid of the two circles. His measures were published in a little work entitled ' Pinax Observationum Chorographicarum.'

After Snellius, the next contribution to the problem came from one of our forgotten philosophers. At the beginning of 167 I Richard Townley, becoming interested in the subject, propounded it as a "chorographical problem." The word chorographical is now replaced by topographical, with little apparent advantage except euphony, the former having a broader and, one would imagine, a more correct signification in survey. Townley's proposition was solved by John Collins, an Oxford bookseller, whose solution appears in the Philosophical Transactions for 167 I. Collins made a real contribution to the subject, for his solution is geometrically the neatest possible, since it necessitates the use of one circle only and an auxiliary point. We shall describe it later. Where Collins' method is employed in English text-books, I have found no reference to his name.

The next name which appears in connection with the problem is that of Laurence Pothenot. Since it would appear that he made no new contribution to the subject, and was in any case anticipated by Snellius and Collins, not to mention the ancient philosophers, it is obvious that the almost universal identification of his name with the problem on the Continent has sprung merely from ignorance of its history. Now that it has been proved for thirty years that he has no valid title, we find his name beginning to appear in English text-books, otherwise excellent.

The trigonometrical solution by aid of an auxiliary angle, now usually employed, was published by Burckhardt in r8or. The cotangent formula 
is of doubtful origin; as all these trigonometrical solutions are fundamentally the same, one form quickly suggests another.

The very practical method of solving the problem by means of an approximate value of one of the unknown angles was first published in the 'Auxiliary Tables of the Survey of India.' In addition to the ordinary observations by theodolite, it demands merely a magnetic bearing of one of the lines, taken by trough or other compass. With this addition the solution is the most rapid possible.

The only instrument in special use in connection with resection is the station-pointer, an English invention apparently. By its means points can be plotted with fair accuracy. In this country its use is practically confined to hydrographers, since observations made on board ship, where a plane-table would be useless, can thus be quickly charted; in fact, at sea the station-pointer fulfils much the same function as the plane-table on land.

We shall now proceed with the first problem. In Fig. I the point resected is shown in several positions. If we consider one of the measured angles, say $\gamma$, then the Snellius point is without the circumscribed circle of the triangle when $\gamma$ is less than $C$ or greater than $180^{\circ}+$ $C$. When $\gamma=C$ or $180^{\circ}+C$, the point lies somewhere on the circumscribing circle, and, as is well known, its position cannot then be fixed. We shall therefore speak of the circumscribing as the "Danger circle," because, even though the point may not be exactly on the circle, its general proximity to the circle renders the location unsatisfactory. If however the point lies near the danger circle, but at the same time near one of the fixed stations, the location may be fairly good. Again when $\gamma$ is $180^{\circ}$, the point lies on the base $\mathrm{AB}$. If $\gamma$ lies between $C$ and $180^{\circ}+C$, the point is within the danger circle; it is moreover within the triangle if $\gamma$ lies between $C$ and $180^{\circ}$, provided that one of the other angles, say $\beta$, lies between $B$ and $180^{\circ}$. This analysis then suggests that if $\gamma$ is round about $90^{\circ} \pm C$, the Snellius point will be well removed from the danger circle, except in the neighbourhood of the fixed stations A, B. This leads us to Fig. 3 .

In Fig. 3 three circles are seen described on the sides $\mathrm{BC}, \mathrm{CA}, \mathrm{AB}$, which we shall always describe as $a, b, c$ respectively. These circles have

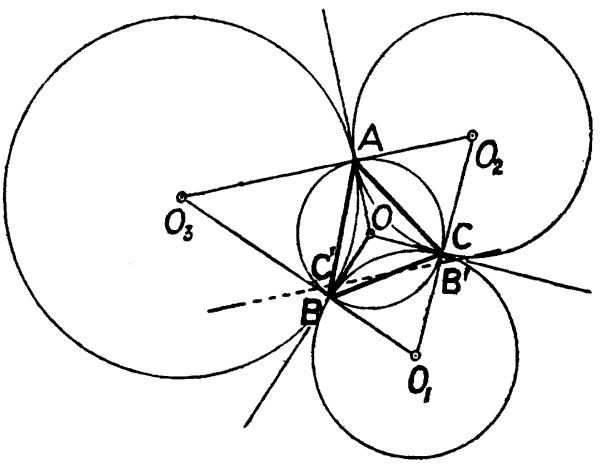

FIG. 3. respectively the chordal angles $90^{\circ} \pm A, 90^{\circ} \pm B, 90^{\circ} \pm C$; that is to say, the angle subtended by $\mathrm{BC}$ at any point of its circle (whose centre is $\mathrm{O}_{1}$ ) 
as either $90^{\circ}+A$ or $90^{\circ}-A$. Now it is easily shown that these three circles touch one another at the apices $\mathrm{A}, \mathrm{B}, \mathrm{C}$ of the fixed triangle, and that they all cut the danger circle orthogonally. We may say then, with a permissible stretch of imagination, that a point moving on any of them endeavours to keep as far away from the danger zone as possible. Still it must cross the danger zone twice on each circle; but, since these crossings are at fixed stations, where no danger can arise within the absolute limits of the station,-a somewhat theoretical conception, and since, in any case,-as is much more important,- no one would employ resection within the purlieus of the fixed stations, these latter may be correctly designated "Neutral points." We shall subsequently see that there may be neutral points in a figure in addition to the fixed stations.

We shall therefore name the three circles $\mathrm{O}_{1}, \mathrm{O}_{2}, \mathrm{O}_{3}$ the "Clear" or "Safe circles." Within the three-rayed star between them, if we clip off the cusps of the rays, resection will be absolutely safe. Outside the danger circle the clear circles give some indication of the limits beyond which the Snellius point should not be placed; but this rule must be used with reservation, for if the angle $\mathrm{C}$, for example, were $90^{\circ}$, the circle $\mathrm{O}_{3}$ would become the line $\mathrm{AB}$, and obviously one could not work along $\mathrm{AB}$ indefinitely.

There is a simple construction for the centres of the clear circles. On $\mathrm{AB}$ mark off $\mathrm{AC}^{\prime}=\mathrm{AC}$, and on $\mathrm{AC}, \mathrm{AB}^{\prime}=\mathrm{AB}$. Then the line $\mathrm{O}_{2} \mathrm{O}_{3}$ is parallel to $\mathrm{B}^{\prime} \mathrm{C}^{\prime}$. Similarly for the other lines of centres.

In Fig. 2 there is shown a common geometrical method of determining the Snellius point. The two angles observed being $\alpha, \beta$, draw CE, making the angle $\mathrm{BCE}=90^{\circ}-\alpha$ with $a$, and $\mathrm{CF}$ making the angle $90^{\circ}-\beta$ with b. If either (or both) of the two values $90^{\circ}-\alpha, 90^{\circ}-\beta$ is negative, the corresponding line (or lines) must then be drawn outside the fixed lines. At $\mathrm{B}$ and $\mathrm{A}$ erect perpendiculars $\mathrm{BE}, \mathrm{AF}$ to $a, b$ respectively. Then with the middle points of $\mathrm{CE}, \mathrm{CF}$ as centres describe circles; these cut in the required point $P$. These two fundamental circles-used at least from the time of Hipparchus-will be mentioned as the "Observation Circles." It will be seen that EPF is one right line, at right angles to CP. LM, which occurs in a later figure, has been drawn parallel, and therefore equal to $\mathrm{EF}$.

There will evidently be a good location of $\mathrm{P}$ when the two observation circles cut at right angles. $\mathrm{ECF}=\mathrm{LPM}$ is then $90^{\circ}$, and $\alpha+\beta$ is then $270^{\circ}-C$; in other words, $\gamma=90^{\circ}+C$. This could have been seen on Fig. 3, for if $\mathrm{P}$ is anywhere on the circle $\mathrm{O}_{3}$, then the two circles $\mathrm{PBC}$, $\mathrm{PCA}$ cut at right angles. This is again the condition for good position suggested by Fig. I. In this case the angles PAC, PBC, which are intimately associated with the solution of the figure, are together equal to a right angle; PMC, PLC are evidently the same angles.

In Fig. 4, are shown variations of the Collins figure and the solution by a single circle. At $\mathrm{A}$ draw the line $\mathrm{AC}^{\prime}$ making the angle $\alpha$ 
with $A B$, and at $B$ the line $\mathrm{BC}^{\prime}$ making the angle $\beta$ with $\mathrm{BA}$. Note that the line from $A$ to $C^{\prime}$ should be drawn in the same general sense as the line from $\mathrm{P}$ to $\mathrm{B}$, and the line from $\mathrm{B}$ to $\mathrm{C}$ in the same sense as the line from $\mathrm{P}$ to $\mathrm{A}$. The point so determined, $\mathrm{C}^{\prime}$, is the Collins auxilliary point. Join $\mathrm{CC}^{\prime}$ and describe a circle round $\mathrm{ABC}^{\prime}$, cutting $\mathrm{CC}^{\prime}$ in the point $\mathrm{P}$, the point required.

It will be noticed that the Collins circle is the "observation circle" corresponding to the angle $\gamma$, which is considered as not

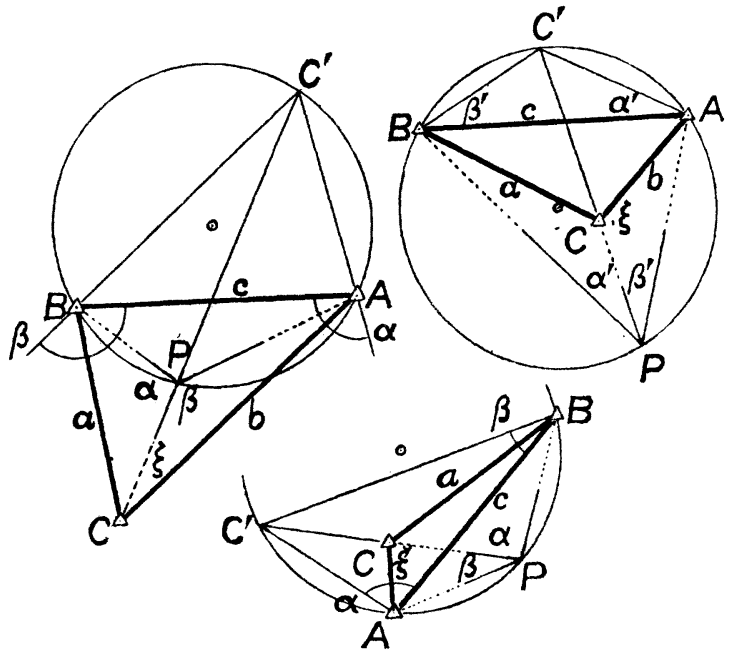

FIG. 4 . observed. The figures indicate also with clearness the impossible case : evidently if the circle through $C^{\prime}$ passes through $C$ also, the point $P$ cannot be fixed $-\mathrm{a}$ conclusion so obvious that Collins, who distinguishes five cases of this problem, probably considered it not worth mentioning. In the impossible case, $\mathrm{C}^{\prime}=\mathrm{r} 80^{\circ}-C$; so that the solution is well removed from impossibility when $\mathrm{C}^{\prime}=90^{\circ}-\mathrm{C}$, or $270^{\circ}-\mathrm{C}$, the Collins circle then becoming a clear circle.

The Collins and Snellius points are antithetic; if $\mathrm{P}$ moves to $\mathrm{C}^{\prime}, \mathrm{C}$ moves to $\mathrm{P}$. If $\mathrm{C}^{\prime}$ goes to infinity, $\mathrm{P}$ is the foot of the perpendicular from $\mathrm{C}$ on $\mathrm{AB}$.

By means of the Collins point the Snellius point can be found directly on the plane-table without employing the ordinary method of trial and error and the aid of the compass. Set the alidade on $\mathrm{AB}$, and orient the the table to view (field) B and clamp; then centering the alidade on (table) $\mathrm{A}$, align on ( field) $\mathrm{C}$ and draw the line $\mathrm{AC}^{\prime}$. Next unclamp the table, set the alidade on $\mathrm{BA}$ and clamp on $(f) \mathrm{A}$; then centring on $(t) \mathrm{B}$ view $(f)$ $\mathrm{C}$ again and draw the line $\mathrm{BC}^{\prime}$. Join $\mathrm{CC}^{\prime}$ and unclamp. Lastly, set the alidade on $\mathrm{C}^{\prime} \mathrm{C}$ and clamp on $(f) \mathrm{C}$; then centre on $(t) \mathrm{A}$ and align on $(f) \mathrm{A}$ and draw the line $\mathrm{AP}$, which meets $\mathrm{CC}^{\prime}$ in the required point. Centring on $(t) \mathrm{B}$ and alignment on $(f)$ B gives the line $\mathrm{PB}$ as a check.

This method is due to Bohnenberger and Bessel, and can be employed in other problems involving resection. The fixed triangle may be enlarged or reduced; reduction may be necessary if the Collins point happens to lie off the table. Reduction or extension is accomplished by 
drawing a line parallel to the base $A B$ and employing its intercept between the two sides $\mathrm{CA}, \mathrm{CB}$. If the Collins auxiliary point is to be utilized in field work, it would be a great advantage to extend the lines between the fundamental stations to the margins of the sheet ; the positions of these stations, supplied by the trigonometrical party, are laid down on the sheets while the plane-tabler is in camp, so that the running out of the lines can be done accurately, with very beneficial results as regards the speed and precision of the subsequent field work.

Rankine, in his 'Manual of Civil Engineering,' gives the Collins figure without mentioning the name of the originator. His trigonometrical solution of the same figure is as follows: Having computed $\mathrm{AC}^{\prime}, \mathrm{BC}^{\prime}$ by the rule of sines, solve the triangle $\mathrm{CAC}^{\prime}$ in which the two sides are now known, together with the included angle $\mathrm{CAC}^{\prime}=180^{\circ}-(\alpha-\mathrm{A})$. We thus obtain the angle ACP, marked $\xi$ on the figures; then knowing the side $b$ and the two angles $\beta, \xi$ in the triangle CAP, compute the sides $\mathrm{AP}, \mathrm{CP}$ by the rule of sines. In the triangle $\mathrm{BCP}$ we have also the angles $a$ and $\mathrm{BCP}=\mathrm{C}-\xi$; these with the side $a$ gives $\mathrm{BP}$ and a check on CP.

In working with rectangular co-ordinates the co-ordinates of $\mathrm{C}^{\prime}$ are computed from those of $\mathrm{A}$ or $\mathrm{B}$. Then from the co-ordinates of $\mathrm{C}$ and $C^{\prime}$ there is obtained the azimuth of $\mathrm{CC}^{\prime}$, which, with the known azimuth of $\mathrm{CA}$, gives the angle $\xi$, whence the solution proceeds as above. This method gives the neatest mathematical resolution of the Collins figure.

The figs. 5 are intended for the more common solutions. The writer some years ago rearranged the formula which makes use of the auxiliary angle, putting it in a form which is perhaps more symmetrical

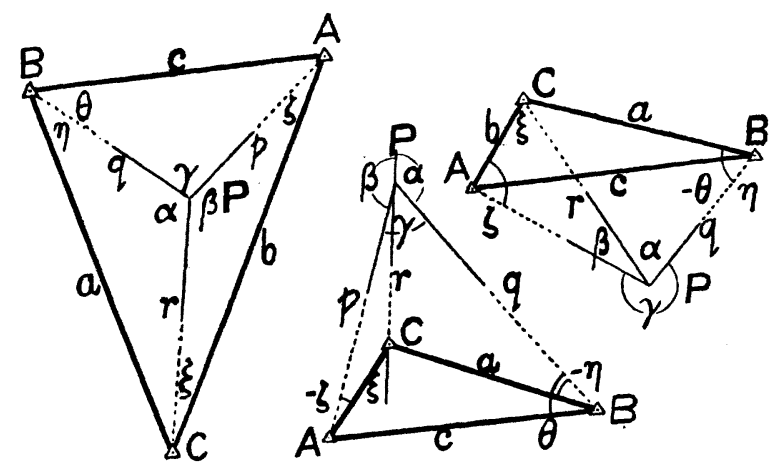

FIG. 5.

and suitable for systematic working. More symbols are introduced than are actually necessary, but the object is to attain greater compactness and symmetry, with adaptation to computation columns. 
Put $l=a: \sin \alpha, \quad m=b: \sin \beta, \quad n=c: \sin \gamma$,

$$
\begin{aligned}
\frac{1}{2}(\alpha-A) & =\alpha_{1}, \\
\tan \left(45^{\circ}+\phi_{1}\right) & =m: n \\
\tan \psi_{1} & =\tan \phi_{1}, \tan \alpha_{1} \\
p=m \sin \left(\alpha_{1}-\psi_{1}\right) & =n \sin \left(\alpha_{1}+\psi_{1}\right) \\
r & =m \sin \left(\alpha_{1}-\psi_{1}+\beta\right), \\
q & =n \sin \left(\alpha_{1}+\psi_{1}+\gamma\right) .
\end{aligned}
$$

These forms are arranged for cyclic interchange; of the three angles $\alpha_{1}, \beta_{1}, \gamma_{1}$ of this interchange, it will be advisable to use that which most nearly approaches $45^{\circ}$. In terms of the angles on the figures, $\alpha_{1}-\psi_{1}=\xi$ and $\alpha_{1}+\psi_{1}=\theta$.

The geometrical interpretation of this formula is shown in Fig. 6, which exhibits a semi-graphical solution of this problem. If three rays $\mathrm{PL}, \mathrm{PM}, \mathrm{PN}$ are drawn from a point $\mathrm{P}$, such that the angle $\mathrm{MPN}=\mathrm{I} 80^{\circ}$ $-(\alpha-A)$, the angle $\mathrm{NPL}=\mathrm{I} 80^{\circ}-(\beta-B)$, and therefore $\mathrm{LPM}=$ $\mathrm{r} 80^{\circ}-(\gamma-C)$, and if on these rays distances equal to $a \operatorname{cosec} \alpha, b \operatorname{cosec} \beta, e$ cosec $\gamma$, are respectively marked off, forming the triangle LMN, then the perpendiculars from $P$ on the sides of this triangle are the required distances from $\mathrm{P}$ to the fixed points, which are the feet of these perpendiculars. The line LM has been already

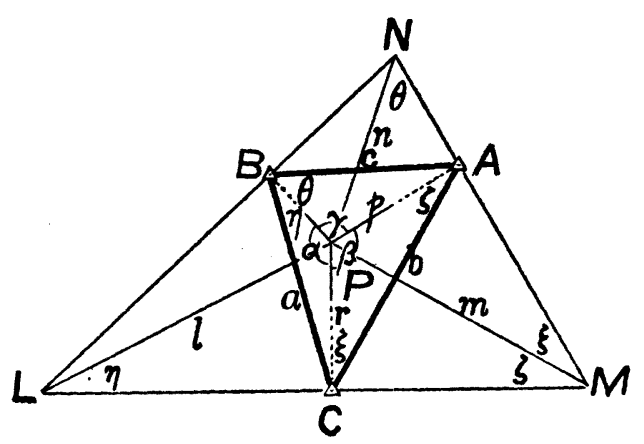

FIG. 6. shown in position in Fig. 2. The accuracy of this construction on paper will exceed that of the tracing-paper or station-pointer method, and the fixed triangle need not be laid down; the method is, in fact, independent of the chart, and the figure can be drawn to the most suitable scale. The slight element of calculation of course adds greatly to its accuracy.

In the author's opinion the most rapid of all mathematical solutions follows from a knowledge of the approximate magnitude of one of the unknown angles of the figure. Such knowledge is obtainable from an observation of magnetic bearing of one of the lines; or the angle may be taken by protractor off a large-scale chart on which the point is laid down by the tracing-paper method; or it may rarely be taken from old measures to a station in the immediate vicinity. If the angle so obtained approximately be $\zeta$ (Fig. 9), then $\eta$ is to be found from $\zeta+\eta=\gamma-C$. Now, if the two triangles BPC, CPA are solved with the rule of sines, by using these two values of $\zeta$ and $\eta$, we have two values of the logarithm of the length of CP. Divide the discrepancy between these two values 
by the sum of the differences for one second of arc in the logarithms of the sines of the angles $\zeta$ and $\eta$ : the result will be the correction in seconds, with appropriate sign, always obvious, to the angle $\zeta$. Correct $\zeta$ accordingly. If the two triangles do not yet give the same value for $\mathrm{CP}$ (in general they will not do so), perform the operation a second time with the amended figures : the results will now usually be concordant.

The method is very simple, and, if the approximation to the unknown angle is quickly obtainable, very rapid, even if the operation has to be performed twice, for we are working along final tabulated lines throughout. For this method Shortrede's tables are excellent, since one should take out the difference of the $\log$ sine with three significant figures. Between $90^{\circ}$ and $180^{\circ}$ the difference of the log sine is of course negative.

The next question which arises is that of the error of the position of the Snellius point corresponding to known or estimated errors in the observed angles. In the case of known errors, we find the displacement of $\mathrm{P}$ as follows : If this displacement be called $\delta \mathrm{P}$, and the errors of $\alpha, \beta$ be $\delta \alpha, \delta \beta$, then in terms of the symbols of Fig. 7 -

$$
\sin ^{2}(\zeta+\eta) \frac{\delta \mathrm{P}^{2}}{r^{2}}=\frac{q^{2}}{a^{2}} \delta \alpha^{2}+\frac{p^{2}}{b^{2}} \delta \beta^{2}+\frac{2 p q}{a b} \cos (\zeta+\eta) \delta \alpha \delta \beta
$$

This expression looks rather formidable, but we shall see that it is capable of geometrical illustration when $\delta a^{2}=\delta \beta^{2}$. To this end it was discovered

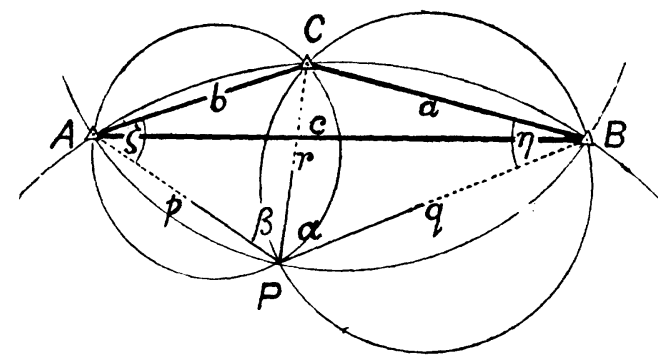

FIG. 7 . that one writer had made use of the principle of inversion, which is applied in Fig. 8. In this figure $\mathrm{A}_{1} \mathrm{~B}_{1} \mathrm{C}_{1}$ is the inverse triangle of $\mathrm{ABC}$ with respect to $\mathrm{P}$ as pole of inversion. Suppose its sides are $a_{1}, b_{1}, c_{1}$, and produce $\mathrm{H}_{1} \mathrm{C}_{1}$ to $\mathrm{A}^{\prime}$ so that $\mathrm{C}_{1} \mathrm{~A}^{\prime}$ $=\mathrm{C}_{1} \mathrm{~A}_{1}=b_{1}$. Join $\mathrm{A}^{\prime} \mathrm{B}_{1}$, which we call $c_{2}$. Then it is found that the displacement of $P$, if $\delta a=+\delta \beta$, is simply $c_{2}$ divided by twice the area of the triangle $\mathrm{A}_{1} \mathrm{~B}_{1} \mathrm{C}_{1}$ and multiplied by a constant (the square of the radius of inversion $k$ ); in other words, the displacement of $\mathrm{P}$ varies inversely as the perpendicular from $C_{1}$ on $c_{2}$. On the other hand, if $\delta \alpha=-\delta \beta$, that is, if the point moves on the Collins circle APB, the displacement of $\mathrm{P}$ varies inversely as the perpendicular on the base $c_{1}$ of the inverse triangle. Not only so, but the direction of the displacement of $\mathrm{P}$ in the former case is given by the supplementary base $c_{2}$, and in the latter case by the direction of the base $c_{1}$ of the inverted triangle. These results are analytically very elegant, and much follows from them. We see at once that the form of the inverse triangle is all-important in considering the error of position 
of $\mathrm{P}$. For example, when $\zeta+\eta=180^{\circ}, \mathrm{P}$ is on the danger circle, as we already know. In this case the inverted triangle degenerates to a straight line, and the perpendicular or its base being therefore zero, the error in the position of $\mathrm{P}$ is theoretically infinite. If the Snellius point is to be well located, the inverse triangle must be well-conditioned. For example, if $\zeta+\eta$ $=90^{\circ}$, the triangle is rightangled, and, as we have already seen, the location of $\mathrm{P}$ will be good. In the special case when

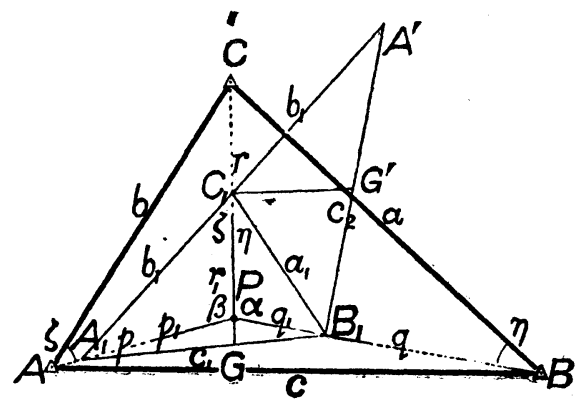

FIG. 8. $\delta \alpha: \delta \beta=a_{1}: b_{1}$, the error is indeterminate : this corresponds to a neutral point.

We next proceed to the more important case of probable or mean square errors, and shall give the preference to the latter, if only for the reason that mean square error presents a less favourable estimate. In this case the formula becomes-

$$
{ }_{2} \Delta \cdot \mu(\mathrm{P})= \pm \mu_{a} k^{2} \sin \mathrm{I}^{\prime \prime} \sqrt{ }\left(a_{1}^{2}+b_{1}^{2}\right)
$$

where $\Delta$ is the area of the inverse triangle, $\mu(\mathrm{P})$ is the mean square error of the position of $\mathrm{P}, \mu_{a}$ is in seconds the m.s.e. of an observed angle, $a_{1}, b_{1}$ are the sides of the inverse triangle, and $k$ is the constant of inversion. We have already given the formula in terms of the lines of the original figure. It is important at this point to distinguish between the measurement of angles and bearings. The formula above supposes that the two angles $\mathrm{BPC}=\alpha$ and $\mathrm{CPA}=\beta$ are observed. We might, however, with equal justice consider that the three bearings $\mathrm{PA}, \mathrm{PB}, \mathrm{PC}$ were measured. The latter is a more symmetrical method of observation, and our inquiry henceforth accordingly resolves itself into two branches following on this necessary division. If bearings are observed the formula becomes-

$$
2 \Delta \cdot \mu(\mathrm{P})= \pm \mu_{b} k^{2} \sin \mathrm{r}^{\prime \prime} \sqrt{ }\left(a_{1}^{2}+b_{1}^{2}+c_{1}^{2}\right)
$$

where $\mu_{b}$ is in seconds the m.s.e. of an observed bearing. In terms of the original lines this formula is-

$$
\mu(\mathrm{P})= \pm \mu_{b} r \sin \mathrm{I}^{\prime \prime}\left(a^{2} p^{2}+b^{2} q^{2}+c^{2} r^{2}\right)^{\frac{3}{2}} / a b \sin (\zeta+\eta)
$$

It is therefore apparent that, remote from the danger circle, the error of position of $\mathrm{P}$ varies roughly as the square of the mean distance from the fixed stations.

These formulæ, then, provide expressions for the displacement of $\mathbf{P}$ corresponding to estimated errors in the angles or bearings, whereby it is possible to determine, with sufficient exactitude, the error of location of $\mathrm{P}$ without resorting to field measurement by other methods. 
These expressions for the error of location of $\mathrm{P}$ enable us to proceed to the last part of our inquiry, namely, the investigation of the best site for P. Dealing first with the case where two angles are observed, we find by analytical methods that, when the error of P's position is a minimum, not only must $\zeta+\eta$ be $90^{\circ}$ or $270^{\circ}$-a condition already frequently mentioned -but in addition $a_{1}$ must equal $b_{1}$; in other words, the inverse triangle must not only be right-angled, but its sides must be equal. This interpreted in symbols of the original figure implies that $p: q=b: a$; that is to say, the ratio of the distances of the Snellius point from the apices A, B of the fixed triangle must be as the adjacent sides. The Snellius point in this case lies on the well-known circle which divides the base internally and externally in the ratio of the sides.

This, then, is a new condition. In some form it crops up in similar investigations in all problems of resection; it may be known as the "condition of equality of position," by which is implied that, if the error of location of a resected point is to be a minimum, the point must not be more
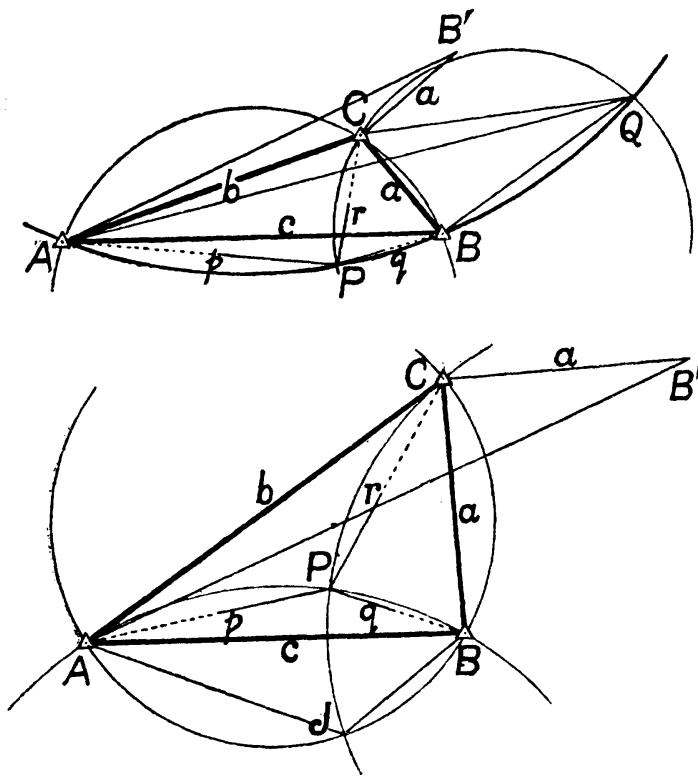

FIG, 9. favourably situated with respect to one fixed point than to another. More generally, in twopoint problems; one resected point must not be more favourably situated than the other with respect to the fixed stations - a principle of wide application.

In Fig. 9, $\mathrm{P}$ is placed at the crossing of the circles which have been found to govern the case of minimum displacement when the two angles are observed. APBQ is the clear circle determined by the condition $\gamma=90^{\circ} \pm \mathrm{C}$; PCQ is the "locus circle," as it will here be shortly named, corresponding to the condition of equality of position. Both circles cut the danger circle at right angles, as might have been anticipated. These two circles cut again in $Q$, which might thus be expected to claim equality with $\mathrm{P}$; but this is not so. With respect to $\mathrm{Q}$ the inverse triangle is equal-sided and rectangular, but its area is much less than that formed from $\mathrm{P}$. $\mathrm{P}$ is then the sole position of minimum displacement. 
The locus circle cuts the danger circle again in $\mathrm{J}$, which is a neutral point.

The position of $\mathrm{P}$ may be determined geometrically in a simple manner. Erect $\mathrm{CB}^{\prime}$ at right angles to $\mathrm{CB}$ and equal to the latter; join $\mathrm{AB}^{\prime}$. Then protract the angle $\mathrm{BAP}$ equal to $\mathrm{B}^{\prime} \mathrm{AC}$ and $\mathrm{ABP}$ equal to $\mathrm{AB}^{\prime} \mathrm{C}$.

The minimum displacement of $\mathrm{P}$ is

$$
\pm \mu_{a} r^{2} \sin \mathrm{I}^{\prime \prime} c / a b
$$

We now come to the more interesting case where the three bearings are observed. Here mathematical analysis proves that for minimum displacement of $\mathrm{P}$ the inverse triangle must be equilateral, and in consequence $p a=q b=r c$. Thus in this case $\mathrm{P}$ is the intersection of three circles, each of which is the locus of an apex of the fixed triangle when the opposite side and the ratio of the adjacent sides are constant. The geometry of the figure is illustrated in Fig. Io. If $\mathrm{O}_{1}, \mathrm{O}_{2}, \mathrm{O}_{3}$ be the centres of the three locus circles, $\mathrm{O}_{1}, \mathrm{O}_{2}, \mathrm{O}_{3}$ are collinear, and if $\mathrm{O}$ is the centre of the danger circle, $\mathrm{O}, \mathrm{P}, \mathrm{Q}$ are collinear ; further, each of the locus circles cuts the danger circle at right angles. These results are well known. Many of the relations of the figure are evident from inversion, since, if two curves cut one another at a certain angle, their inverse curves cut at the same angle. Thus the danger circle inverts into the circle $A_{1} B_{1} C_{1}$, and the locus circles invert into the diameters $A_{1} H_{1}, B_{1} I_{1}, C_{1} J_{1}$; since the diameters cut one another at $60^{\circ}$, the locus circles cut one another at $60^{\circ}$. Again, if the observation circles, which are not drawn in the figure, are considered, it is seen that the circle BCP inverts into the line $\mathrm{B}_{1} \mathrm{C}_{1}$; and similarly the two circles CPA, APB invert into the lines $C_{1} A_{1}, A_{1} B_{1}$. Since these lines both cut one another and the invert of the danger circle at $60^{\circ}$, the observation circles cut one another and the danger circle at $60^{\circ}$. The analogy with a well-conditioned triangle is obvious : the observation circles in this case are all well-conditioned as to their cutting of the danger circle. All the circles mentioned cut all the others at either $30^{\circ}, 60^{\circ}$, or $90^{\circ}$. The observation circles are all clear circles, but not exactly in the sense hitherto used ; they were formerly considered clear if they cut the danger circle at $90^{\circ}$. But only two can cut at $90^{\circ}$; here three are cutting at $60^{\circ}$.

For the same reason as was given in the case of angle observation, Q is not the most favourable position. But $\mathrm{P}$ and $\mathrm{Q}$ are together unique in this, that they are the only two points from which the original triangle inverts into one which is equilateral; $\mathrm{P}$ and $\mathrm{Q}$ are in fact special centres of inversion for the triangle.

$\mathrm{H}, \mathrm{I}, \mathrm{J}$ are all neutral points, and $\mathrm{A}_{1} \mathrm{~J}_{1} \mathrm{~B}_{1} \mathrm{H}_{1} \mathrm{C}_{1} \mathrm{I}_{\mathrm{I}}$ is a regular hexagon. $P$ then occupies precisely the same relation to the triangle $\mathrm{HIJ}$ as to $\mathrm{ABC}$. These neutral points represent points of weakness in the danger zone : in their vicinity the Snellius point may approach nearer to the danger circle 
than elsewhere; in other words, a point moving off the danger circle in their vicinity improves its position, as regards resection, with maximum rapidity. The command of position from the neutral points is not, however, equally strong ; for example, the Snellius point would have to move farther away from $J$ than from I to secure equally good location. If the fixed triangle, bel inverted from J, say, it will be found that the triangle becomes a line bisected by JC.

Fig. Io shows a geometrical construction for $\mathrm{P}$. On $\mathrm{AB}$ describe an equilateral triangle $\mathrm{ABC}^{\prime \prime}$ and join $\mathrm{CC}^{\prime \prime}$. Draw the angle $\mathrm{BCP}$ equal to $\mathrm{ACC}^{\prime \prime}$ and $\mathrm{CBP}$ equal to $\mathrm{AC}^{\prime \prime} \mathrm{C}$, thus obtaining $\mathrm{P}$. Or we may find $\mathrm{P}$ by

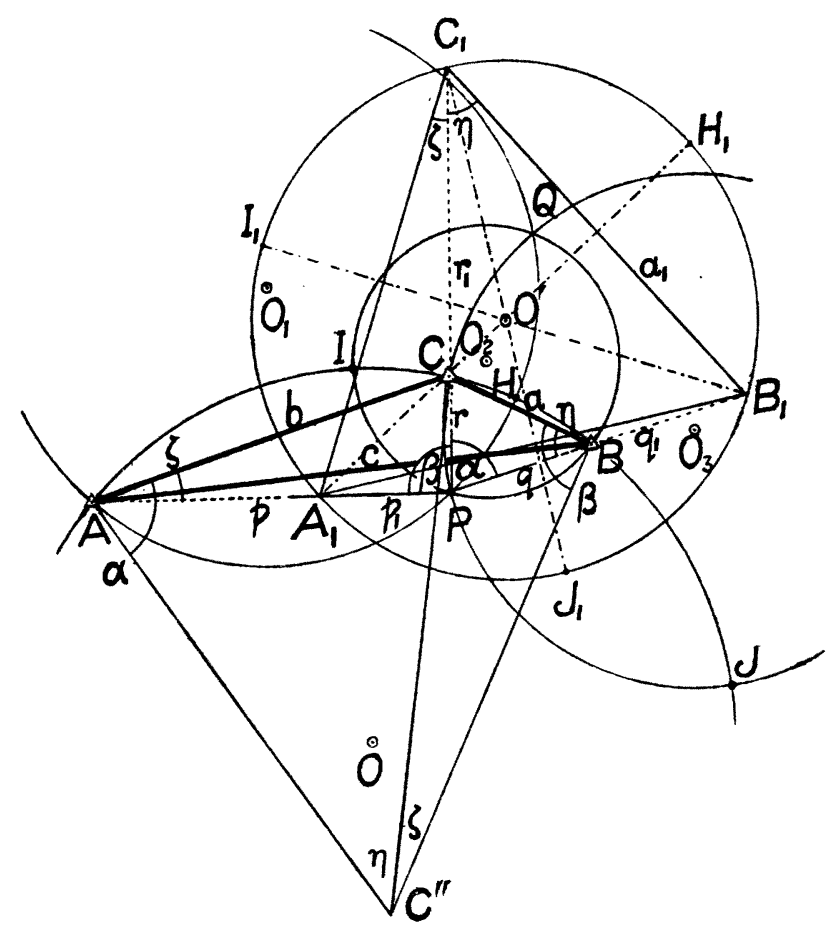

FIG. 10.

the method of Fig. 9. Draw CB, in this case making an angle $\mathrm{BCB}^{\prime}=60^{\circ}$; the remainder of the construction is as before. In this case the Snellius point in its best position is on the base when $\mathrm{C}=120^{\circ}$; in the former case, that of angle measurement, it is on the base when $\mathrm{C}=90^{\circ}$.

When bearings, or as they are sometimes termed, directions, are measured, the minimum displacement of $\mathrm{P}$ is $\pm 2 \mu b \sin \mathrm{I}^{\prime \prime} s^{4} / a b c$,

where $s^{2}=a p=b q=c r=a b c / \sqrt{ }\left\{a^{2}+b^{2}-2 a b \cos (60+C)\right\}$ 


\section{The Second Problem.}

Every surveyor in the field is rapidly made acquainted with the difficulty of finding a spot from which a sufficient number of surrounding stations are visible. This is more particularly true of a country broken by deep valleys or numerous ridges. To find connections for points in these valleys or in ridge-divided plains, in which lie as a rule the more valuable areas, is a task which is constantly confronting the observer. It follows, therefore, that it may almost be said of any method of resection that, ceteris paribus, its merit is in inverse proportion to the number of fixed stations required. This statement is more strictly warranted in the case of trigonometrical interpolation than in plane-table work, for it may be said that practically the plane-table demands a minimum of three stations.

The present method, involving only two fixed stations, is therefore entitled to second place; although not so generally used as the first, it is usually employed in work of a somewhat higher class. The first method is above all suited to the plane-table ; the second is not directly applicable to the plane-table, but is eminently adapted for the theodolite. It has been so largely used in India, particularly in the Himalayan and transfrontier region, and more especially in the deep valleys of this region, that it may be known as the Indian Problem.

In Fig. I I, A, B are the two fixed stations and $\mathrm{P}, \mathrm{S}$ two points resected from them, the observed angles therefore being as marked $\gamma, \mu, \gamma^{\prime}, \mu^{\prime}$.

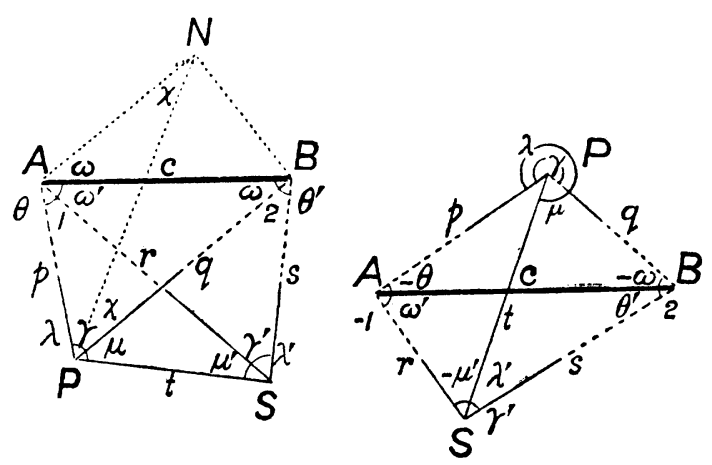

FIG. II. Now from these angles we can compute the ratio $\mathrm{PB}: \mathrm{PA}$, or as marked $q: p$. Hence in the triangle APB there are known the angle $\mathrm{APB}=\gamma$ and the ratio of the adjacent sides; wherefore the base angles $\mathrm{ABP}=\omega$ and $\mathrm{BAP}=\theta$ can be computed. Major R. A. Wahab, R.E., used an auxiliary angle $\chi$, with the following geometrical construction: Draw AN parallel to $\mathrm{PB}$, and drop $\mathrm{BN}$ perpendicular to $\mathrm{AN}$. Then the angle ANP or BPN is the angle $\chi$. Having computed $\chi$ he thence derives $\omega,=\mathrm{BAN}$ or $\mathrm{ABP}$. Knowing $\omega$, or any one of the angles at the fixed stations, we thence derive all the other angles of the figure, whence the lengths of the lines are derived by the ordinary rule of sines.

Here again it is apparent that the solution depends on that of a triangle in which one angle and the ratio of the adjoining sides is known - the same difficulty which revealed itself in the case of the first problem. 
The various artifices which are used arise from the simple fact that these data in the triangle do not permit of pure logarithmic computation without supplementary aid. Here again, then, the auxiliary angle $\phi$ may be employed. If we put

$$
\begin{gathered}
\cot (\phi+45)=p: q=\sin \mu^{\prime} \operatorname{cosec}\left(\gamma^{\prime}+\mu^{\prime}\right) \operatorname{cosec} I \sin 2 \\
\tan \left(\omega+\frac{1}{2} \gamma\right)=\tan \frac{1}{2} \gamma \tan \phi
\end{gathered}
$$

then

This gives $\omega$, from which the lengths of the lines follow as before.

Perhaps the most obvious solution is that by Colonel T. F. B. RennyTailyour, R.E. It is immediately apparent that the geometrical solution is merely a question of scale, since, if the length PS were known, the figure could be immediately plotted. Accordingly the geometrical solution entails merely the adoption of a provisional length for PS, the plotting of the corresponding figure by means of the observed angles, and the enlargement or reduction of the figure in the proportion of the true length of $\mathrm{HB}$ to the length obtained from such a plotment.

This principle is easily applied trigonometrically. Assume that PS is one foot or one metre (according to the units employed), and compute the corresponding length of $\mathrm{AB}$. Then add the difference between the logarithm of the length thus computed and the true logarithm to the logarithms of the lengths of all the computed lines; the result is the solution of the figure.

It is not to be supposed that this method shortens the trigonometrical computation. We have here to solve the reverse problem, that of finding the length of $\mathrm{AB}$ when the two points $\mathrm{A}_{1} \mathrm{~B}$ are intersected from $\mathrm{P}, \mathrm{S}$; and this solution again involves the cosine formula, that is to say, the formula for the solution of a triangle given two sides and the included angle. We may note in passing that this problem of intersection is known as Hansen's on the Continent. Hansen's paper was published in I84I; since, however, the solution was given for the resection by "a Member of the Philosophical Society of Oxford" (whose name cannot be found) in the Philosophical Transactions for $\mathrm{I} 685$, it is obvious that Hansen has no claim to originality.

The problem may be simply solved by means of an approximate value of one of the angles, say $\omega$. Let the value of $p$, thence computed directly from the triangle $\mathrm{ABP}$, be $p^{\prime}$, and that similarly computed through the the triangles ABS, ASP be $p^{\prime \prime}$; then

$$
\Delta \omega=\left(\log p^{\prime}-\log p^{\prime \prime}\right) /\{\nabla(\omega)-\nabla(2+\omega)\}
$$

where $\Delta \omega$ is the error in seconds of the approximation to $\omega$, and $\nabla(\omega)$, $\nabla(2+\omega)$ are respectively the differences for one second in the log sine of the angles $\mathrm{ABP}=\omega$ and $\mathrm{ABS}=\mathbf{2}+\boldsymbol{\omega}$. Perform the operation twice if necessary.

The displacement of either resected point corresponding to known or mean square errors of the observed angles is calculated without much difficulty, but will not be given here. 
The position of minimum displacement, corresponding to a common error in the observed angles, is almost obvious. Since the measured angles are placed symmetrically in the figure, it is apparent that neither $\mathrm{P}$ nor $\mathrm{S}$ can occupy a preferential position with respect to the fixed stations $A, B$. We can here invoke our principle of "equality of position." It follows that the figure for minimum displacement must be rectangular and symmetrical. The two forms are shown in Figs. 12, where it is almost obvious that the crossed form must give the better fixing for a given length of $\mathrm{AB}$.

This problem is not directly applicable to the plane-table, as heretofore remarked. If the solution be
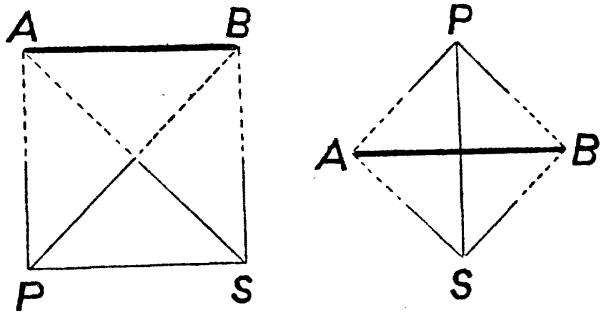

FIG. 12.

desired on the plane-table, it is necessary to adopt a provisional base, which may be chosen to represent as closely as can be estimated the line between the resected points in length and direction. Working from the two stations, we thus obtain a figure which must be corrected in dimensions and orientation to agree with the line between the fixed stations.

The PRESIDENT: I regret the absence of our Chairman this afternoon. Dr. Mill is still suffering from his recent accident, and I fear he will not be available for some little time. We are to have an address this afternoon on a highly technical subject from a gentleman who is particularly well qualified to deal with it, for Mr. McCaw has had much experience of geodetic survey in various parts of the world, especially in South Africa and on the UgandaCongo boundary.

\section{(Mr. McCaw then read the paper printed above, and a discussion followed.)}

Lieut.-Colonel Lyons, R.E.: I am glad this question has been treated by Mr. McCaw, because there are many of these investigations on surveying which do not come before surveyors in this country as often as they might, and especially those that set forth clearly the effect of the errors which must occur and the necessity for taking them into account in selecting a method. I hope that, when the paper is published, Mr. McCaw will give fully the references to those papers by Townley and Bessel and others that he has quoted, for, as he says, they are not generally known in this country, although they are quoted freely in the principal text-books on the Continent. The history of the development of surveying is very interesting, as the case which $\mathrm{Mr}$. McCaw cites has shown, but the whole subject well deserves to be followed up more thoroughly than has been done of late years in this country. Mr. McCaw says that resection is not applicable or would not usually be used in cadastral survey, but perhaps he is using the word "cadastral" in a limited sense, because resection and somewhat elaborate methods of applying it are very generally used in the cadastral surveys of Europe; for instance, where it is necessary to obtain co-ordinates of such resection points for use in the further development of a survey of landed property. Such a cadastral survey is one 